\title{
Emerging Clinical Evidence on Online Hemodiafiltration: Does Volume of Ultrafiltration Matter?
}

\author{
Bernard Canaud $^{\mathrm{a}, \mathrm{b}}$ Sudhir K. Bowry ${ }^{\mathrm{a}}$ \\ ${ }^{a}$ Medical Board EMEALA, Fresenius Medical Care, Bad Homburg, Germany; ${ }^{b}$ Montpellier University I, \\ Medical School, Montpellier, France
}

\section{Key Words}

End-stage chronic kidney disease $\cdot$ Renal replacement therapy · Hemodiafiltration · Convective volume · Patient outcome

\begin{abstract}
Online hemodiafiltration (OL-HDF), first described in 1985, is today a widely prescribed treatment modality for end-stage chronic kidney disease (CKD) patients. Other than in the United States, prescription of the treatment modality is widespread with a steady increase since its inception. Indeed, in Western Europe, more CKD patients receive OL-HDF than peritoneal dialysis, hitherto the second most prescribed therapy after conventional hemodialysis. The rise and success of OL-HDF can be attributed to diverse clinical advantages that have been documented over the last two decades. Numerous publications attest to the beneficial effects of OL-HDF in terms of removal of a broad spectrum of uremic toxin, anemia control, phosphate reduction, increased hemodynamic stability and blood pressure control and less dialysis-related amyloidosis, to mention just a few. Significantly, the improvement in these conditions is considered to contribute to improved patient outcomes. Despite the extended worldwide clinical experience, elaborate scientific validation of the principles of the therapy and technical innovations that facilitate its prescription, a point of contention is whether OL-HDF leads to a reduction of mortality rates. A number
\end{abstract}

of observational and retrospective analyses have indicated a survival benefit, while prospective investigations involving small numbers of patients but nevertheless specifically addressing survival have further supplied evidence of improved survival with OL-HDF. The quest for large-scale, multicenter prospective randomized controlled trials examining patient survival led to the CONTRAST and the Turkish OLHDF trials. Both trials have been concluded and published recently. In this chapter, we document and assess the key investigations that have examined the impact of OL-HDF on patient outcome and survival. Based on the findings of previous analyses and of the two recently concluded trials, it appears that the volume of convection appears to be decisive towards the survival benefit accredited to OL-HDF. We consider the implications of this new evidence.

Copyright $\odot 2013$ S. Karger AG, Basel

\section{Introduction}

Online hemodiafiltration (OL-HDF) has gained increasing clinical acceptance over the last two decades, being recognized as a safe, comfortable and more efficient alternative to conventional hemodialysis [1]. The cold sterilization filtration process of dialysis fluids (dialysate and infusate) enabled by online sterilizing filters offers a safe and affordable way of producing large amounts of substitution fluid for HDF modalities [2, 3]. OL-HDF ma-

\section{KARGER \\ Fax +4161306 1234 \\ E-Mail karger@karger.ch}

www.karger.com (c) $2013 \mathrm{~S}$. Karger AG, Basel

0253-5068/13/0353-0055\$38.00/0

Accessible online at:

www.karger.com/bpu
Prof. B. Canaud

Chairman of Medical Board EMEALA, Fresenius Medical Care

1 Else Kröner Strasse

DE-61352 Bad Homburg (Germany)

E-Mail Bernard.Canaud@fmc-ag.com 
chines have the advantages of being certified by European-notified bodies and recognized by EBPG [4-6]. The versatility of online production of dialysis fluids permits to cover adequately all individual patient needs in terms of volume of substitution (metabolic needs), site of substitution as post-, pre- or mixed-dilution (hemorrheologic characteristics) and blood flow limitations $[7,8]$. Automation and technical improvements of OL-HDF machines have considerably simplified their handling and provide the same user confidence and comfort as for standard HD machines. Cost-effectiveness of OL-HDF, which varies from one country to another, depends upon the costs of disposable filters, additional microbiological monitoring and specific hygienic rules requested by individual health authorities to warrant absolute safety of the method [9].

The scientific nephrology community has also underlined that the OL-HDF option offers the best platform for developing renal replacement therapies of the future. By enhancing solute removal capacities and giving unlimited access to quasi-sterile and non-pyrogenic intravenous quality fluid, OL-HDF offers new ways for moving to total automation (e.g. priming, rinsing and feedback volume-controlled systems) and therapy cost-saving [10].

From a clinician's perspective and considering the potential benefits of chronic kidney disease (CKD) patient outcomes, it seems puzzling not to offer OL-HDF as a first-line renal replacement therapy in end-stage CKD [9, 11]. In this chapter, we assess all the investigations that have examined the impact of OL-HDF on patient survival. Based on the findings of previous analyses and of the two recently randomized concluded trials, we consider the implications of this new evidence for the supporting role of OL-HDF in the renal replacement therapy armamentarium.

\section{Substitution of Fluid in Online Hemodiafiltration}

The essence of HDF is the controlled replacement of large volumes of ultrafiltered plasma water by infusion of substitution fluid into the blood of the patient while maintaining a constant dialysate flow $[12,13]$. The ultrafiltration volume is the sum of the infused fluid volume and the volume of fluid that needs to be removed due to weight gain between dialysis treatment sessions. The addition of substitution fluid and its subsequent removal from diluted blood has been shown to be a more efficient means of reducing the levels of various uremic toxins that accumulate in CKD.
Uremia is attributed to the build-up of a large number of solutes of various sizes. There is general consensus that both small and large uremic retention solutes, commonly referred to as uremic toxins, need to be removed during renal replacement therapy to enhance patient well-being [14]. A number of treatment strategies have been developed to achieve this objective. A fundamental approach to enable the removal of larger uremic solutes was the development of high-flux dialysis membranes having sufficiently high mean pore sizes to allow large solutes to pass through yet restrict the leakage of useful substances beyond a certain size range [15]. By adding and removing substitution fluid in conjunction with the use of high-flux membranes, a more efficient means of removing a broad spectrum of uremic toxins is achieved. This approach incorporates the convective transport (passage of solutes following fluid flow by ultrafiltration across high-permeability membranes, also known as 'solvent drag') component, in addition to the diffusive transport (based on the difference in concentration between the blood and dialysis fluid compartments) mechanism for the removal of solutes $[16,17]$. Dialysis machines with highly reliable volumetric control and balancing systems ensure risk-free fluid balance and management of simultaneous fluid reinfusion and ultrafiltration.

Although the essentials of conventional HDF (involving infusion of defined volumes of sterile substitution fluid from bags) have been validated since the 1970s, the availability of unlimited quantities of cheap, high-purity (microbiologically) replacement fluids initially restricted the application of the therapy to a wider patient population $[18,19]$. The advent in 1985 of 'online' preparation of non-pyrogenic substitution fluid from dialysis fluid fulfilled these limitations, paving the way to an entirely new era of more efficient blood detoxification [20, 21]. New technologies and concepts have evolved since this landmark to facilitate the delivery of large volumes of highly pure substitution fluids during OL-HDF.

There are two main variants of OL-HDF, depending on the site of administration of substitution fluid in the extracorporeal circuit. When substitution fluid enters the circuit before the dialyzer in predilution HDF, blood is diluted before solute transfer mechanisms occur across the semipermeable membrane. When substitution fluid is administered after the dialyzer (postdilution OL-HDF), ultrafiltered fluid volume is essentially replaced. The choice between pre- or postdilution depends mainly on considerations of blood rheology and filtration fraction of individual patients. 


\section{Clinical Experience with Online Hemodiafiltration: Focus on Patient Survival}

Since 1985, the number of CKD patients treated with OL-HDF has risen steadily. At the end of 2011, worldwide $4.9 \%$ of the HD patients were treated with the OL-HDF modality, taking into account that no OL-HDF is in use in the USA which constitutes around $20 \%$ of the world's HD population. In Western Europe, $18 \%$ of the HD population is treated with OL-HDF, with countries such as Switzerland, Slovenia and Slovakia having over $60 \%$ of their patients on OL-HDF [1].

The growth in the prescription of OL-HDF is attributed to a number of clinical benefits as perceived by both patients and nephrologists. Among the main documented conditions and pathways positively affected by the therapy are: (a) enhanced removal of larger uremic toxins; numerous studies have verified significant reduction of $\beta_{2^{-}}$ microglobulin $\left(\beta_{2}-\mathrm{MG}\right)$, a recognized surrogate of the middle molecules and also involved in diverse pathological conditions [22-24]; (b) better control of anemia, with a reduction of erythropoietin dosage [25-28]; (c) a more effective removal of phosphate and control of hyperphosphatemia [29-31]; (d) improved lipid profiles [32, 33]; (e) reduced inflammation and oxidative stress [34-37]; (f) lower incidence of side effects, hypotensive episodes and hospitalization [38, 39], and ( $\mathrm{g}$ ) better patient well-being and quality of life: recovery of nutritional status, increased appetite and feeling of being more energetic [40, 41].

The essential facts and figures regarding the study type, design and objectives of the main clinical studies on OL-HDF examining patient outcomes are summarized in table 1 . The investigations include retrospective analyses, prospective observational as well as prospective small-scale (e.g. single-center) assessment of patient mortality rates with OL-HDF. The key findings and conclusions (outcomes) of each of these studies are also listed in table 1. Varying objectives, study design or methodology (parameters assessed and statistics) make this body of evidence highly heterogeneous but nevertheless represents a highly valuable indicator of the clinical efficiency and efficacy of OL-HDF. Detailed assessment of the value as well as limitations of these investigations have been reviewed and published [41].

Thus, the extensive clinical experience acquired over almost three decades has established OL-HDF as an advanced and beneficial renal replacement therapy modality in routine practice. Significantly, no clinical incidences or studies indicating any detrimental aspects of the OL-HDF therapy option have been published.

Recent Findings of Randomized

Controlled Trials in OL-HDF
The CONTRAST and Turkish OL-HDF Studies: LargeScale Prospective Randomized Controlled Trials Examining the Impact of OL-HDF on Patient Survival

Despite the acknowledged clinical advantages accredited to OL-HDF, a wider prescription of the modality has been held back by the call for more conclusive evidence in the form of large-scale, prospective randomized clinical trials to demonstrate a survival benefit for OL-HDF [42]. As indicated in table 1, the issue of patient survival has been already been addressed for some time with a number of studies suggesting reduced patient mortality when patients are treated with OL-HDF. However, as this evidence indicating a survival benefit with OL-HDF has been derived from observational or retrospective analyses from a small patient population, the need for larger, multicenter clinical trials has been frequently emphasized to strengthen the case for OL-HDF. Consequently, two such trials, the CONTRAST and the Turkish OL-HDF studies, were undertaken and their results published recently. Table 2 summarizes the salient features of the two studies.

The Turkish HDF Study (table 2) that compared OLHDF with high-flux hemodialysis (HF-HD) in terms of mortality and morbidity likewise found no differences between the two groups with respect to the primary endpoint (composite of all-cause mortality and non-fatal cardiovascular event rate) [43]. In the post hoc analysis, treatment with substitution volumes over the median of 17.4 l/session (high-efficiency OL-HDF) was associated with a significant risk reduction of both overall and cardiovascular mortality.

The CONTRAST Study (table 2), comparing survival rates between low-flux dialysis and postdilution OLHDF, did not reveal differences between the two treatment modes in terms of the primary endpoint, namely all-cause mortality [44]. Further, there were no significant differences between the two groups in the frequency and incidence of fatal and non-fatal cardiovascular events. However, post hoc analyses again showed a significant inverse relationship between delivered convection volume and mortality risk. After correction for potential confounding factors, the hazard ratio for all-cause mortality was considerably lower in the group of patients treated with the highest delivered convective volumes (>21.95 liters).

Despite the fact that the CONTRAST and the Turkish OL-HDF studies were randomized controlled trials, both studies present some significant pitfalls that contribute to reduce strength of the evidence. As partially addressed by 
Table 1. Clinical trials and studies on OL-HDF

\begin{tabular}{|c|c|}
\hline $\begin{array}{l}\text { Locatelli et al. } \\
{[39]}\end{array}$ & $\begin{array}{l}\text { Open-label RCT/LF-HD vs. predilution OL-HDF vs. predilution OL-HDF/27 centers (Italy); } 146 \text { patients/2-year follow-up. } \\
\text { Endpoint: intradialytic hypotension. } \\
\text { - Unlike conventional HD, convective therapies (HDF and HF) reduce ISH (primary endpoint) in long-term dialysis patients. }\end{array}$ \\
\hline Schiffl [56] & $\begin{array}{l}\text { Unblinded, prospective cross-over RCT/ultrapure HF-HD vs. OL-HDF/1 center (Germany); } 76 \text { patients/4-year follow-up. } \\
\text { Endpoint: mortality, diverse biochemical indices, quality of life. } \\
\text { - OL-HDF is a safe, effective and well-tolerated therapy for end-stage renal disease patients even in the long run. } \\
\text { - Both HF-HD and OL-HDF were associated with sustained reductions of pretreatment } \beta_{2} \text {-MG levels, the decrease being greater } \\
\text { with OL-HDF. }\end{array}$ \\
\hline $\begin{array}{l}\text { Wizeman et al. } \\
\text { [49] }\end{array}$ & $\begin{array}{l}\text { Prospective RCT/LF-HD vs. OL-HDF/1 center (Germany); } 44 \text { patients/2-year follow-up. Endpoint: morbidity, mortality, } \beta_{2}-\mathrm{MG} \\
\text { as marker of uremic toxicity, hypotension, anemia. } \\
\text { - } \quad \text { No difference for morbidity, mortality, blood pressure, hypotensive episodes, hematocrit and EPO dose. } \\
\text { - } \text { OL-HDF ensures an excellent dialysis quality which apparently takes time to translate into measurable clinical sequelae. }\end{array}$ \\
\hline
\end{tabular}

Locatelli et al. High-efficiency LF-HD vs. HF-HD vs. HDF/71 centers (Italy); 380 patients/24-month follow-up. Endpoint: polysulfone vs. [57] cuprophan, HF-HD vs. HDF.

- Significant decrease in predialysis $\beta_{2}-\mathrm{MG}$ in HF-HD and HDF could have a clinical impact considering that its accumulation and amyloidosis are important long-term dialysis-related complications.

Tiranathanagul Prospective, observational, non-randomized/HF-HD vs. predilution OL-HDF/1 center (Thailand); 22 patients/3-year follow-up. et al. [51] Endpoint: adequacy, biochemical parameters.

- For OL-HDF, decreased incidence of hypotension, improved nutritional status and patient well-being, well-controlled lipid profile, normal C-reactive protein maintained, elevated removal of ( $\left.\beta_{2}-\mathrm{MG}\right)$.

Vliar et al. Retrospective observational comparative analysis/HF-HD vs. OL-HDF/1 center (UK); 858 patients/18-year period. Endpoint: [58] survival, anemia management, nutrition, blood pressure.

- Improved mortality with OL-HDF compared with HF-HD: treatment solely with HDF had a hazard for death of 0.66 compared with patients treated solely with HF-HD.

- No benefits of HDF over HF-HD with respect to anemia management, nutrition, mineral bone metabolism or blood pressure control.

Panichi et al. Prospective, observational/LF-HD vs. HF-HD vs. HDF/15 centers (Italy); 757 patients/30-month follow-up. Endpoint: all-cause

[59] and cardiovascular mortality, inflammation markers.

- HDF was associated with an improved cumulative survival independent of the dialysis dose.

- Synergic effect of CRP and proinflammatory cytokines as the strong predictors of all-cause and cardiovascular mortality.

Vinhas et al. Prospective, observational/HF-HD vs. OL-HDF/1 center (Portugal); 329 patients/3-year follow-up. Endpoint: all-cause mortality, [60] hospitalization relative risk, biochemical parameters.

- Annual crude mortality rate decreased from 19.9 deaths/100 patient-years (HF-HD) to 8.9 deaths/100 patient-years (HDF period); survival attributed to reduction of cardiovascular mortality.

- Relative risk for hospitalization was not significantly reduced for patients on HDF compared to those on HD.

Canaud et al. Observational/LF-HD vs. HF-HD vs. low- and high-efficiency OL-HDF/multicenter (France, Germany, Italy, Spain, UK); 2,165

[61] patients/ 3-year follow-up. Endpoint: mortality rates.

- Patients on high-efficiency HDF had a significant 35\% lower mortality risk than those on LF-HD.

- Patients on HDF had a higher likelihood of having albumin $>4 \mathrm{~g} / \mathrm{dl}$ and a lower likelihood of ferritin $>800 \mathrm{ng} / \mathrm{dl}$ than LF-HD.

Jirka et al. Prospective, epidemiological, observational/HD vs. HDF/multicenter (Czech Republic, Hungary, Italy, UK); 2,564 patients/1-year [62] follow-up. Endpoint: mortality risk.

- After adjustment for age, gender, comorbidities and time on renal replacement therapies, mortality risk reduction was $35.5 \%$ with HDF compared to HD.

Bosch et al. Prospective, observational/high-efficiency LF-HD vs. HF-HD vs. HDF/1 center (USA); 183 patients/6-year study. Endpoint:

$[63]$

survival.

- Kaplan-Meier survival curves of all patients (i.e. including all 3 modalities) were compared with those from USRDS data.

- Standardized mortality data (SMR), derived for each high-efficiency treatment modality, showed significance only for HDF (relative to USRDS data), indicating better survival for this modality.

Locatelli et al. Historical, prospective/HD vs. HDF vs. hemofiltration/44 centers; 6,444 patients/follow-up till 30 months. Endpoint: mortality [64] and amyloid-related amyloidosis.

- Trend towards better survival: the relative risk for mortality adjusted for age, gender and comorbidities was $10 \%$ lower in patients treated with HDF and hemofiltration.

- Adjusting for age and diabetic status, the relative risk for carpal tunnel syndrome surgery was $42 \%$ lower in patients treated with HDF and hemofiltration (compared to standard HD). 
Table 2. The Turkish OL-HDF and CONTRAST studies

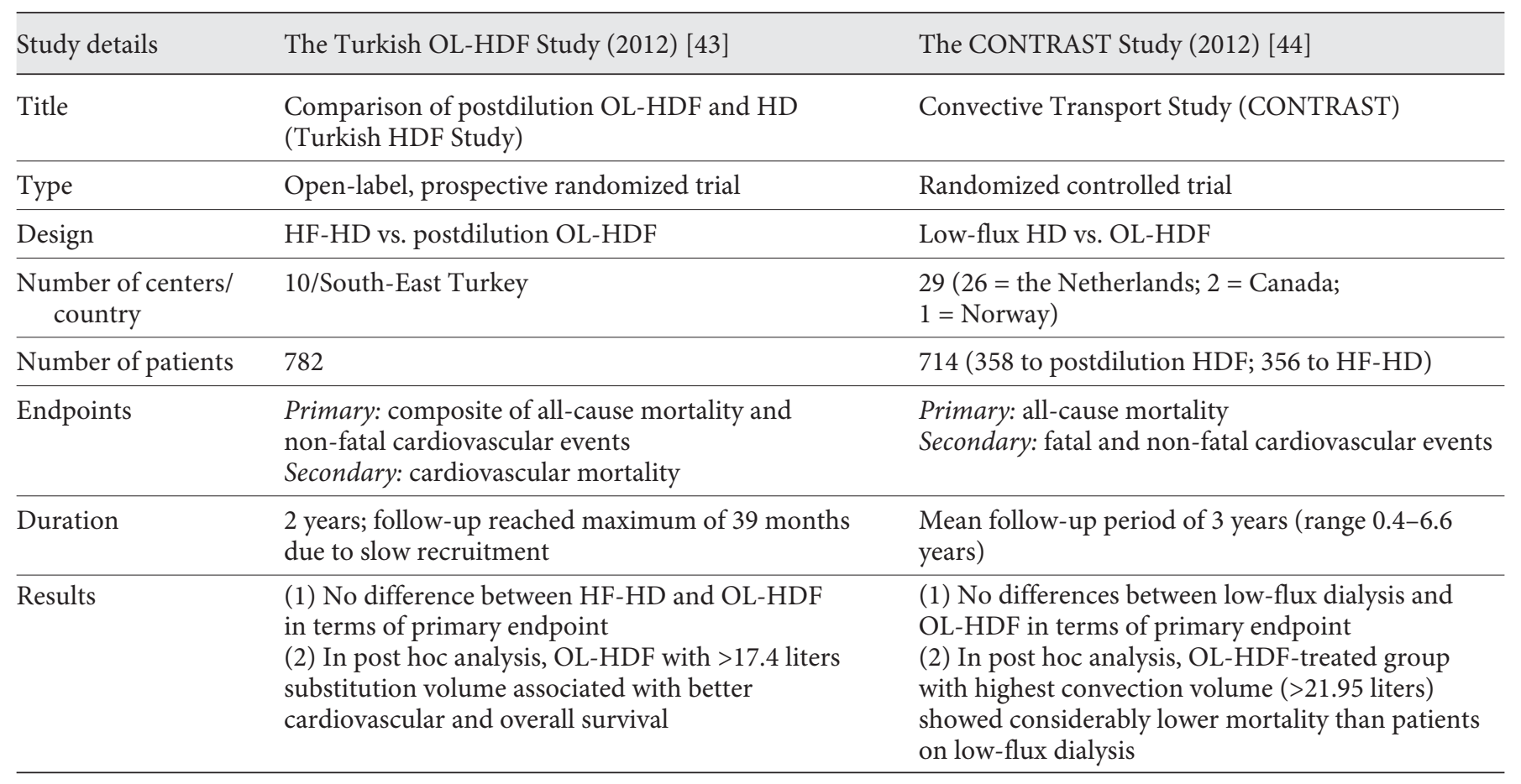

Kuhlmann [45] in the editorial accompanying the CONTRAST Study, ultrafiltration volume targeted in the prespecified study protocol (5-6 liters/h) was achieved only in one-third of the dialysis patients. In addition, large variations of ultrafiltration volumes were noted from center to center (range 13-22 liters/session) underlining the gap between prescription and treatment delivery. Factors impeding the capacity of achieving the prescribed ultrafiltration volume have been clearly identified by Penne et al. [46]. For a fixed treatment duration time, they rely on a poor blood flow, high albumin concentration and high hematocrit [46]. As a matter of fact, one can argue that targeted ultrafiltration volume was not adequately achieved in the majority of patients, a situation of protocol violation that may invalidate primary objectives of both studies.

Interestingly, the findings of both the CONTRAST and the Turkish HDF studies are consistent with the DOPPS data which first suggested the link between convection dose and favorable patient outcomes [47]. Although the DOPPS results were based on observational analysis, the findings were based on the study of a large patient population in four different countries, thereby taking into account differences between therapy practices. A survival benefit was described for high-efficiency
OL-HDF ( $>15$ liters/session substitution volume, excluding net ultrafiltration for weight loss).

Three separate studies thus suggest that the volume of convection imparts a survival benefit for dialysis patients in terms of reducing mortality rates. Two of the studies reach this conclusion based on subgroup analyses and one on observational data. Despite such limitations, each study involved a large patient population and was carried out independently in different countries. The emerging trend that the exchange volume is possibly a decisive criterion of patient survival with OL-HDF is consistent with both the current understanding of uremic toxicity and the mechanisms involved in the detoxification of blood with dialysis therapies.

\section{Convective Volume - A New Paradigm of High-Efficiency Therapies?}

In order to judge the efficacy and the potential clinical benefits of OL-HDF, it is essential to use a quantifying indicator of the convective clearance. Following the recommendations of the EUDIAL group, it has been proposed to use the total volume of ultrafiltration achieved per HDF session adjusted to postdilution mode for the 
various dilution modalities (pre-, mixed- or mid-HDF). This is an easy and clinically relevant surrogate indicator of the convective component. Alternatively, it is proposed to assess more precisely the biological effect by dosing biomarkers to reflect the convective action of the HDF. Among uremic toxins, $\beta_{2}-\mathrm{MG}$, a $12-\mathrm{kDa}$ peptide, seems to be one of the most representative and clinically relevant representatives of the middle molecule uremic toxins and strongly implicated in both morbidity and mortality of CKD patients. Although it may be too early to set some predialysis (or time-average concentration) target values for circulating $\beta_{2}-\mathrm{MG}$ concentration, it is interesting to note that in a post hoc analysis of the HEMO study, Cheung et al. [48] have identified that predialysis serum $\beta_{2}$-MG concentrations $>27.5 \mathrm{mg} / \mathrm{l}$ were associated with an increased risk of death in a dose-dependent manner. Indeed, the HEMO study has established that the relative risk of death accounting for confounding factors was $11 \%$ higher per each $10-\mathrm{mg} / \mathrm{l}$ increase of predialysis $\beta_{2}-\mathrm{MG}$ concentrations based on the threshold value of $27.5 \mathrm{mg} / 1$ in HD patients. From a clinical perspective, until more precise data are provided, the threshold value of $27.5 \mathrm{mg} / 1$ concentration of $\beta_{2}$-MG could be taken as a risk reference indicator for HD patients [48]. In this perspective, it is worth noting that studies analyzing predialysis $\beta_{2}-\mathrm{MG}$ concentrations in OL-HDF-treated patients are highly concordant, reporting values comprised between 22 and $25 \mathrm{mg} / \mathrm{l}[23,49-52]$.

It is attractive to suggest that the application of high exchange volumes are the sum of diverse clinical practices applied over the last two decades to refine and enhance convective therapies. From a recent per-protocol analysis of the CONTRAST Study, it has been shown that the volume of substitution that can be achieved is a summation of patient-related factors, medical prescription components, nursing practices and technically related factors. Among components that condition the success of delivering the optimal therapy in HDF, it is interesting noting that four groups of factors interact in unison and condition deliverability of the suitable convective dose: (a) some are related to the patient characteristics including the vascular access flow deliverable, the total protein concentration (protocrit) and the hematocrit; (b) some depend on the OL-HDF prescription and in this case the duration of session is determinant; (c) some are conditioned by facility nursing practices including anticoagulation regimen and protocol as well as quality care of achieving best blood flow and OL-HDF targets, and (d) some are dependent on technical features of the OL-HDF machine and fluid management, setting the priority ei- ther on the ultrafiltration flow or the transmembrane pressure limits. It is not our intent to detail the ways of ensuring the optimal volume of ultrafiltration here, but simply to underline the fact that findings of the aforementioned OL-HDF studies cannot be interpreted without some form that is adjusted to the volume of ultrafiltration achieved per session (or per week) for each individual patient [53].

The recent findings of the two large-scale randomized controlled trials (CONTRAST and Turkish OL-HDF studies) have reinforced the concept of convective dose originated by the cohort DOPPS study [47]. Based on recent clinical findings, it is now tempting to speculate that the threshold total convective volume to bring a significant beneficial effect to CKD patient outcome should be in the range of 19-22 liters/session in postdilution HDF mode. To satisfy more closely patient metabolic needs, the convective volume may be tailored and expressed as liters per kilogram body weight $(70-80 \mathrm{ml} / \mathrm{kg} / \mathrm{h})$ or body surface area $\left(2,000-2,500 \mathrm{ml} / \mathrm{m}^{2} / \mathrm{h}\right)$. When different HDF modalities are used (e.g. predilution or mixed dilution) to facilitate the application of HDF in problematic patients (e.g. poor blood flow, hemorheologic difficulties), an appropriate dilution factor should additionally be accounted for in calculating the effective convective volume delivered to the patient.

\section{Conclusion}

Based on the findings of previous cohort studies and of the two recently reported randomized controlled trials (CONTRAST and Turkish OL-HDF), one can conclude that the volume of convection matters. It may represent a decisive component of the survival benefit for OL-HDFtreated patients. From a statistical viewpoint, the two recent interventional trials will be registered as negative studies not achieving their primary objective of reducing all-cause and cardiovascular mortality in dialysis patients. Again, from a methodological viewpoint, one should note that a targeted convective volume was achieved in a minority of patients (one third) and interestingly only these patients had significant survival benefits. From a purely clinical perspective, it would be unfortunate to deprive end-stage CKD patients from deriving advantages from a superior renal replacement therapy due simply to suboptimal care practices and methodological considerations $[54,55]$.
Canaud/Bowry 


\section{References}

$>1$ Sichart JM, Moeller S: Utilization of hemodiafiltration as treatment modality in renal replacement therapy for end-stage renal disease patients: a global perspective. Contrib Nephrol 2011;175:163-169.

-2 Canaud B, Bosc JY, Leray H, Stec F: Microbiological purity of dialysate for on-line substitution fluid preparation. Nephrol Dial Transplant 2000;15(suppl 2):21-30.

$\checkmark 3$ Canaud B, Bosc JY, Leray-Moragues H, Stec F, Argiles A, Leblanc M, Mion C: On-line haemodiafiltration. Safety and efficacy in long-term clinical practice. Nephrol Dial Transplant 2000;15(suppl 1):60-67.

4 Polaschegg HD, Roy T: Technical aspects of on-line hemodiafiltration. Contrib Nephrol 2007;158:68-79.

5 Kümmerle W: Facing regulatory challenges of on-line hemodiafiltration. Contrib Nephrol 2011;175:74-80.

-6 European Best Practice Guidelines Expert Group on Hemodialysis, European Renal Association. Section IV. Dialysis fluid purity. Nephrol Dial Transplant 2002;17(suppl 7):45-62.

$>7$ Canaud B, Lévesque R, Krieter D, Desmeules S, Chalabi L, Moragués H, Morena M, Cristol JP: On-line hemodiafiltration as routine treatment of end-stage renal failure: why pre- or mixed dilution mode is necessary in on-line hemodiafiltration today? Blood $\mathrm{Pu}$ rif 2004;22(suppl 2):40-48.

$>8$ Potier J: Mid-dilution: an innovative highquality and safe haemodiafiltration approach. Contrib Nephrol 2007;158:153-160.

$\checkmark 9$ Blankestijn PJ, Ledebo I, Canaud B: Hemodiafiltration: clinical evidence and remaining questions. Kidney Int 2010;77:581-587.

10 Canaud B, Granger A, Chenine-Khoualef L, Patrier L, Morena M, Leray-Moragués H: On-line hemodialysis monitoring: new tools for improving safety, tolerance and efficacy; in Azar AT (ed): Modeling and Control of Dialysis Systems: Biofeedback Systems and Soft Computing Techniques of Dialysis. Berlin, Springer, 2012, vol 2, pp 775-809.

11 Henderson LW: Dialysis in the 21st century. Am J Kidney Dis 1996;28:951-957.

$\checkmark 12$ Henderson LW, Colton CK, Ford CA: Kinetics of hemodiafiltration. II. Clinical characterization of a new blood cleansing modality. J Lab Clin Med 1975;85:372-391.

-13 Leber HW, Wizemann V, Goubeaud G, Rawer P, Schutterle G: Hemodiafiltration: a new alternative to hemofiltration and conventional hemodialysis. Artif Organs 1978;2:150-153.

14 Vanholder R, Baurmeister U, Brunet P, Cohen G, Glorieux G, Jankowski J; European Uremic Toxin Work Group: A bench to bedside view of uremic toxins. J Am Soc Nephrol 2008; 19:863-870.
15 Bowry SK: Membrane requirements for high-flux and convective therapies. Contrib Nephrol 2011;175:57-68.

16 Sprenger KB, Kratz W, Lewis AE, Stadtmüller U: Kinetic modeling of hemodialysis, hemofiltration, and hemodiafiltration. Kidney Int 1983;24:143-151.

17 Locatelli F, Manzoni C, Di Filippo S: The importance of convective transport. Kidney Int Suppl 2002;80:115-120.

18 Henderson LW, Beans E: Successful production of sterile pyrogen-free electrolyte solution by ultrafiltration. Kidney Int 1978; 14: 522-525.

19 Henderson LW, Sanfelippo ML, Beans E: On-line preparation of sterile pyrogen-free electrolyte solution. Trans Am Soc Artif Intern Organs 1978;24:465-467.

20 Canaud B, N'Guyen QV, Lagarde C, Stec F, Polaschegg HD, Mion C: Clinical evaluation of a multipurpose dialysis system adequate for hemodialysis or for postdilution hemofiltration/hemodiafiltration with on-line preparation of substitution fluid from dialysate. Contrib Nephrol 1985;46:184-186.

21 Canaud B: The early years of on-line HDF: How did it all start? How did we get here? Contrib Nephrol 2011;175:93-109.

$>22$ Lornoy W, Becaus I, Billiouw JM, Sierens L, Van Malderen P, D’Haenens P: On-line haemodiafiltration. Remarkable removal of $\beta_{2^{-}}$ microglobulin. Long-term clinical observations. Nephrol Dial Transplant 2000;15 (suppl 1):49-54.

23 Ward RA, Schmidt B, Hullin J, et al: A comparison of on-line hemodiafiltration and high-flux hemodialysis: a prospective clinical study. J Am Soc Nephrol 2000;11:23442350.

24 Pedrini LA, De Cristofaro V, Comelli M, Casino FG, Prencipe M, Baroni A, Campolo G, Manzoni C, Colì L, Ruggiero P, Acquistapace I, Auriemma L. Long-term effects of high-efficiency on-line haemodiafiltration on uraemic toxicity. A multicentre prospective randomized study. Nephrol Dial Transplant 2011;26:2617-2624

25 Vaslaki L, Major L, Berta K, Karatson A, Misz M, Pethoe F, Ladanyi E, Fodor B, Stein G, Pischetsrieder M, Zima T, Wojke R, Gauly A, Passlick-Deetjen J: On-line haemodiafiltration versus haemodialysis: stable haematocrit with less erythropoietin and improvement of other relevant blood parameters. Blood Purif 2006;24:163-173.

26 Locatelli F, Manzoni C, Del Vecchio L, Di Filippo S, Pontoriero G, Cavalli A: Management of anemia by convective treatments. Contrib Nephrol 2011;168:162-172.

$>27$ Bowry SK, Gatti E: Impact of hemodialysis therapy on anemia of chronic kidney disease: the potential mechanisms. Blood Purif 2011; $32: 210-219$
28 Panichi V, Scatena A, Paoletti S, Migliori M: Impact of dialysis technique on renal anemia. Contrib Nephrol 2011;171:261-265.

29 Lornoy W, De Meester J, Becaus I, Billiouw JM, Van Malderen PA, Van Pottelberge M: Impact of convective flow on phosphorus removal in maintenance hemodialysis patients. J Ren Nutr 2006;16:47-53.

30 Davenport A, Gardner C, Delaney M; Pan Thames Renal Audit Group: The effect of dialysis modality on phosphate control: haemodialysis compared to haemodiafiltration. The Pan Thames Renal Audit. Nephrol Dial Transplant 2010;25:897-901.

-31 Penne EL, van der Weerd NC, van den Dorpel MA, Grooteman MP, Lévesque R, Nubé MJ, Bots ML, Blankestijn PJ, ter Wee PM; CONTRAST Investigators: Short-term effects of on-line hemodiafiltration on phosphate control: a result from the randomized controlled Convective Transport Study (CONTRAST). Am J Kidney Dis 2010;55: 77-87.

32 Wanner C, Bahner U, Mattern R, Lang D, Passlick-Deetjen J: Effect of dialysis flux and membrane material on dyslipidaemia and inflammation in haemodialysis patients. Nephrol Dial Transplant. 2004;19:25702575.

33 Schiffl H, Lang SM: Effects of dialysis purity on uremic dyslipidemia. Ther Apher Dial 2010;14:5-11.

-34 Ramírez R, Martín-Malo A, Aljama P: Evolution of the concept of biocompatibility and the cardioprotective effect of on-line hemodiafiltration. Contrib Nephrol 2011;175:110 116.

35 Panichi V, Tetta C: On-line hemodiafiltration in the large RISCAVID study. Contrib Nephrol 2011;175:117-128.

36 Filiopoulos V, Hadjiyannakos D, Metaxaki P, Sideris V, Takouli L, Anogiati A, Vlassopoulos D: Inflammation and oxidative stress in patients on hemodiafiltration. Am J Nephrol 2008;28:949-957.

>37 Calò LA, Naso A, Davis PA: Hemodiafiltration and oxidative stress in dialysis patients. Blood Purif 2009;28:59-60.

-38 Mion M, Kerr PG, Argiles A, Canaud B, Flavier JL, Mion CM: Haemodiafiltration in high-cardiovascular-risk patients. Nephrol Dial Transplant 1992;7:453-454.

39 Locatelli F, Altieri P, Andrulli S, Bolasco P, Sau G, Pedrini LA, Basile C, David S, Feriani M, Montagna G, Di Iorio BR, Memoli B, Cravero R, Battaglia G, Zoccali C: Hemofiltration and hemodiafiltration reduce intradialytic hypotension in ESRD. J Am Soc Nephrol 2010;21:1798-1807.

40 Masakane I: Choice of modality with the use of high-performance membrane and evaluation for clinical effects. Contrib Nephrol 2011;173:84-94. 
-41 Gatti E, Ronco C: Seeking an optimal renal replacement therapy for the chronic kidney disease epidemic: the case for on-line hemodiafiltration. Contrib Nephrol 2011;175:170185.

42 Canaud B, Morena M, Leray-Moragues H, Chalabi L, Cristol JP: Overview of clinical studies in hemodiafiltration: what do we need now? Hemodial Int 2006; 10(suppl 1):S5-S12.

43 OkE, Asci G, Sevinc-Ok E, Kircelli F, Yilmaz M, Hur E, Sezis Demirci M, Ozdogan O, Demirci C, Onen Sertoz O, Duman S, Ozkahya M, Kayikcioglu M, Elbi H, Basci A, Toz $\mathrm{H}$ : Comparison of postdilution on-line hemodiafiltration and hemodialysis (Turkish HDF Study). Nephrol Dial Transplant 2012, in press.

-44 Grooteman MP, van den Dorpel MA, Bots ML, Penne EL, van der Weerd NC, Mazairac $\mathrm{AH}$, den Hoedt $\mathrm{CH}$, van der Tweel I, Lévesque R, Nubé MJ, ter Wee PM, Blankestijn PJ; CONTRAST Investigators: Effect of on-line hemodiafiltration on all-cause mortality and cardiovascular outcomes. J Am Soc Nephrol 2012;23:1087-1096.

45 Kuhlmann MK: On-line hemodiafiltration: not a self-fulfilling prophecy. J Am Soc Nephrol 2012;23:974-975.

-46 Penne EL, van der Weerd NC, Bots ML, van den Dorpel MA, Grooteman MP, Lévesque R, Nubé MJ, Ter Wee PM, Blankestijn PJ; CONTRAST Investigators: Patient- and treatment-related determinants of convective volume in post-dilution haemodiafiltration in clinical practice. Nephrol Dial Transplant 2009;24:3493-3499.

47 Canaud B, Bragg-Gresham JL, Marshall MR, Desmeules S, Gillepsie BW? Depner T, Klassen $\mathrm{P}$, Port F: Patients receiving hemodiafiltration or hemofiltration have lower mortality risk than patients receiving hemodialysis without replacement fluid in Europe: The Dialysis Outcomes and Practice Patterns Study (DOPPS). J Am Soc Nephrol 2004;14: $31 \mathrm{~A}$.
48 Cheung AK, Greene T, Leypoldt JK, Yan G, Allon M, Delmez J, Levey AS, Levin NW, Rocco MV, Schulman G, Eknoyan G; HEMO Study Group: Association between serum $\beta_{2}$-microglobulin level and infectious mortality in hemodialysis patients. Clin J Am Soc Nephrol 2008;3:69-77.

49 Wizemann V, Lotz C, Techert F, Uthoff S: On-line haemodiafiltration versus low-flux haemodialysis. A prospective randomized study. Nephrol Dial Transplant 2000;15 (suppl 1):43-48.

50 Oates T, Pinney JH, Davenport A: Haemodiafiltration versus high-flux haemodialysis: effects on phosphate control and erythropoietin response. Am J Nephrol 2011;33:70-75.

51 Tiranathanagul K, Praditpornsilpa K, Katavetin $P$, Srisawat N, Townamchai N, Susantitaphong P, Tungsanga K, Eiam-Ong S: Online hemodiafiltration in Southeast Asia: a three-year prospective study of a single center. Ther Apher Dial 2009;13:56-62.

52 Susantitaphong P, Tiranathanagul K, Katavetin $\mathrm{P}$, Hanwiwatwong $\mathrm{O}$, Wittayalertpanya S, Praditpornsilpa K, Tungsanga K, EiamOng S: Efficacy comparison between simple mixed-dilution and simple mid-dilution online hemodiafiltration techniques: a crossover study. Artif Organs 2012, E-pub ahead of print.

53 Penne EL, van Berkel T, van der Weerd NC, Grooteman MP, Blankestijn PJ: Optimizing haemodiafiltration: tools, strategy and remaining questions. Nephrol Dial Transplant 2009;24:3579-3581.

54 Canaud BJ: Changing paradigms of renal replacement therapy in chronic kidney disease patients: ultrapure dialysis fluid and highefficiency hemodiafiltration for all? Kidney Int 2009;76:591-593.
Locatelli F, Canaud B: Dialysis adequacy today: a European perspective. Nephrol Dial Transplant 2012;27:3043-3048.

56 Schiffl H: Prospective randomised crossoverlong-term comparison of on-line haemodiafiltration and ultrapure haemodialysis. Eur J Med Res 2007;12:26-33.

57 Locatelli F, Mastroangelo F, Redaelli B, et al: Effects of different membranes and dialysis technologies on patient tolerance and nutritional parameters. Kidney Int 1996;50:12931302.

58 Vliar E, Fry AC, Wellsted D, et al: Long-term outcomes in on-line hemodiafiltration and high-flux dialysis: a comparative analysis. Clin J Am Soc Nephrol 2009;4:1944-1953.

-59 Panichi V, Rizza GM, Paoletti S, et al: Chronic inflammation and mortality in hemodialysis: effect of different renal replacement therapies. Results from the RISCAVID study. Nephrol Dial Transplant 2008;23: 2337-2343.

60 Vinhas J, Vaz A Barreto C, et al: Survival advantage of patients on haemodiafiltration is independent of dialysis dose and patient characteristics: data from a single centre. Port J Nephrol Hypert 2007;21:287-292.

61 Canaud B, Bragg-Gresham JL, Marshall MR, et al: Mortality risk for patients receiving hemodiafiltration versus hemodialysis. European results from the DOPPS. Kidney Int 2006;69:2087-2093.

62 Jirka T, Cesare S, Di Benedetto A, et al: Mortality risk for patients receiving hemodiafiltration versus hemodialysis. Kidney Int 2006;70:1524.

63 Bosch JP, Lew SQ, Barlee V, et al: Clinical use of high-efficiency hemodialysis treatments: long-term assessment. Hemodial Int 2006; 10:73-81.

64 Locatelli F, Marcelli D, Conte F, et al: Comparison of mortality in ESRD patients on convective and diffusive extracorporeal treatments. Kidney Int 1999;55:286-293. 